\title{
HIPERVOLEMIA MAIS TRÍPLICE OCLUSÃO VASCULAR NO TRATAMENTO DA LESÃO TRAUMÁTICA DA VEIA CAVA RETRO-HEPÁTICA E VEIAS HEPÁTICAS
}

\author{
HYPERVOLEMIA AND TRIPLE VESSEL OCCLUSION IN THE TREATMENT \\ OF TRAUMATIC RETROHEPATIC VENA AND HEPATIC VEIN INJURIES
}

\author{
Wilson Luiz Abrantes, TCBC-MG
}

\section{INTRODUÇÃO}

A lesão da veia cava retro-hepática (VCRH) e das veias hepáticas (VH) simboliza o grande desafio do cirurgião do trauma. Dificuldades de acesso à lesão, perda sangüínea intensa (sangramento anterógrado e retrógrado) alterações hemodinâmicas graves, improvisações e inexperiência cirúrgica com este tipo de lesão explicam a mortalidade de $30 \%$ a $90 \%$ entre os dois terços dos pacientes que conseguem chegar ao hospital. $\mathrm{O}$ isolamento vascular do fígado é fundamental para o controle da lesão. $\mathrm{O}$ isolamento pode ser feito por procedimentos complexos (shunt interno), por métodos sofisticados, incompatíveis com hospitais públicos (circulação extracorpórea) e pela tríplice oclusão vascular (TOV) (oclusão do pedículo hepático, veia cava inferior acima das veias renais e no interior saco pericárdico) (Figura 1). A TOV é um procedimento técnico simples. Ao contrário dos dois outros métodos, causa redução do retorno venoso e queda do débito cardíaco de $40 \%$ a $50 \%$. Este inconveniente contraindica sua aplicação em pacientes hipovolêmicos e/ou chocados (parada cardíaca). O reconhecimento da eficiência e simplicidade da TOV como método de isolamento vascular tem levado alguns autores a empregá-la com a clampagem simultânea da aorta com o objetivo de controlar o estado hemodinâmico ${ }^{1}$. A manobra não é isenta de riscos.

O objetivo do presente trabalho é demonstrar que a TOV precedida do estado de hipervolemia é um procedimento seguro para o controle da lesão venosa. A técnica foi empregada com sucesso em três pacientes.

\section{TÉCNICA}

A descrição da técnica será feita em etapas distintas para melhor valorizar a sequiência e importância de cada uma delas.
1. Controle temporário da hemorragia: é feito mediante compressão da lesão com o objetivo de evitar qualquer perda sangüínea. A baixa pressão do sistema venoso contribui para a eficácia da manobra. A compressão será mantida até a conclusão da terceira etapa.

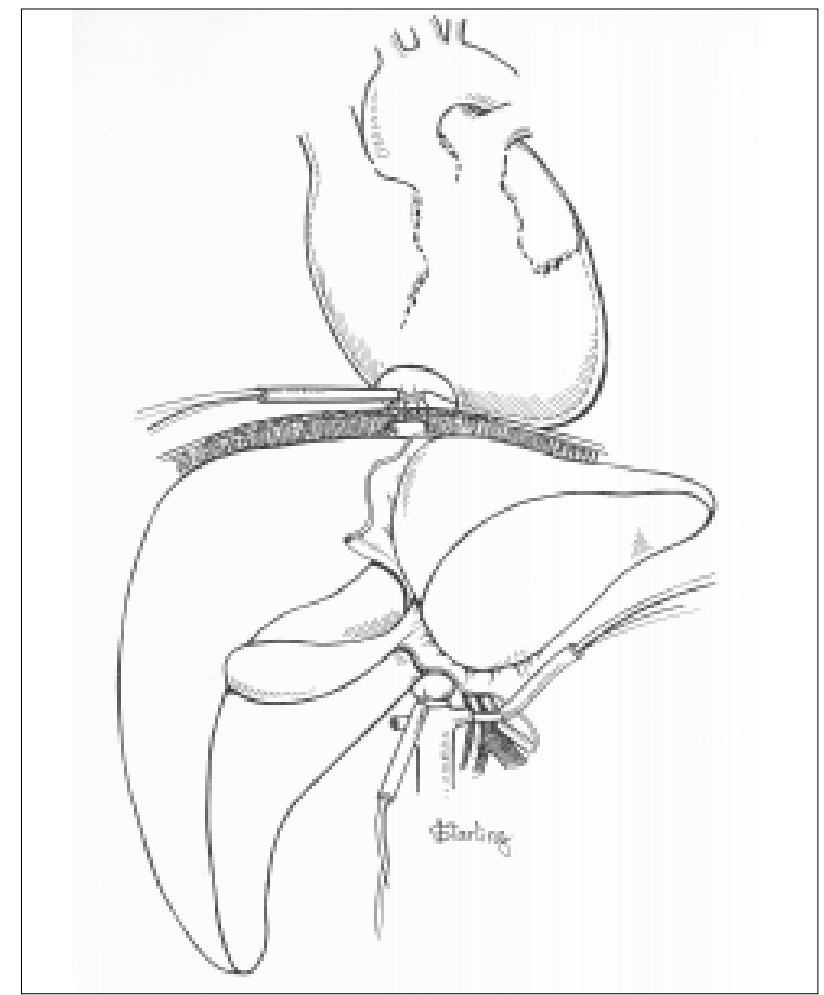

Figura 1 - Tríplice oclusão vascular (pedículo hepático VCI acima das veias renais e interior pericárdio).

\section{Chefe Cirurgia do Hospital João XXIII}

Recebido em 24/07/2000

Aceito para publicação em 03/04/2001

Trabalho realizado no Hospital João XXIII da Fundação Hospitalar do Estado de Minas Gerais (FHEMIG) 
2. Restauração da volemia normal (reanimação): simultaneamente com o controle temporário da hemorragia, cabe ao anestesista o restabelecimento da volemia, mantendo monitorização adequada e prevenindo a hipotermia. Durante essa fase é feita a esternotomia e o preparo dos vasos a serem ocluídos (cadarço na VCI acima das veias renais, no interior do pericárdio e dos elementos do pedículo hepático). Do ponto de vista prático, a normalização da pressão intra-arterial (PIA), a presença de tensão na parede da aorta e enchimento da VCI são indícios de volemia normal.

3. "Adiantamento da volemia"; "pré-carga"; "reforço ou expansão de volemia": todos estes termos indicam uma volemia adicional necessária para atingir o estado de hipervolemia. Consiste em infundir rapidamente 1.500 a $2.000 \mathrm{ml}$ de líquidos na circulação do paciente. A hipervolemia assim obtida é condição fundamental para evitar as complicações hemodinâmicas secundárias à TOV (redução do retorno venoso e queda do débito cardíaco e arritmias, parada cardíaca).

4. TOV: é realizada após o estabelecimento da hipervolemia e na seguinte seqüência: tríade portal (manobra de Pringle), veia VCI acima das renais e no interior do pericárdio.

5. Acesso a VCRH e/ou VH mediante hepatotomia pela técnica de fratura digital a partir da lesão hepática existente. Na ausência dessa será feita na linha de projeção da VCRH sobre o parênquima hepático. Essa projeção fica situada medialmente a VHD.

6. Identificação da lesão, venorrafia e/ou ligadura quando se tratar de uma das VH.

7. Hemostasia rigorosa na área da hepatotomia com ligadura seletiva dos vasos e ductos biliares, seguida de epiploplastia e drenagem.

Resumindo, o tratamento da lesão venosa comporta três fases. Na primeira, o objetivo é alcançar o estado de hipervolemia mediante oclusão temporária da lesão e reposição agressiva de líquido. Na segunda, é feita a TOV para manter o campo operatório exangue para a realização da terceira fase: hepatotomia, venorrafia e/ou ligadura venosa (Figura 2).

\section{DISCUSSÃO}

Na técnica proposta, a seqüência e o momento de se proceder à TOV estão bem definidos, o que não ocorre na literatura. Nesta, a falta de definição é responsável pelos insucessos do método. De fato, prevalecem na literatura expressões vagas e imprecisas como: "a oclusão (TOV) é realizada após a "estabilização" do paciente, ou após "reanimação" ou "ressuscitação" ou "simultaneamente com a oclusão é feita reposição agressiva de líquidos". "Estabilização", "reanimação", res-

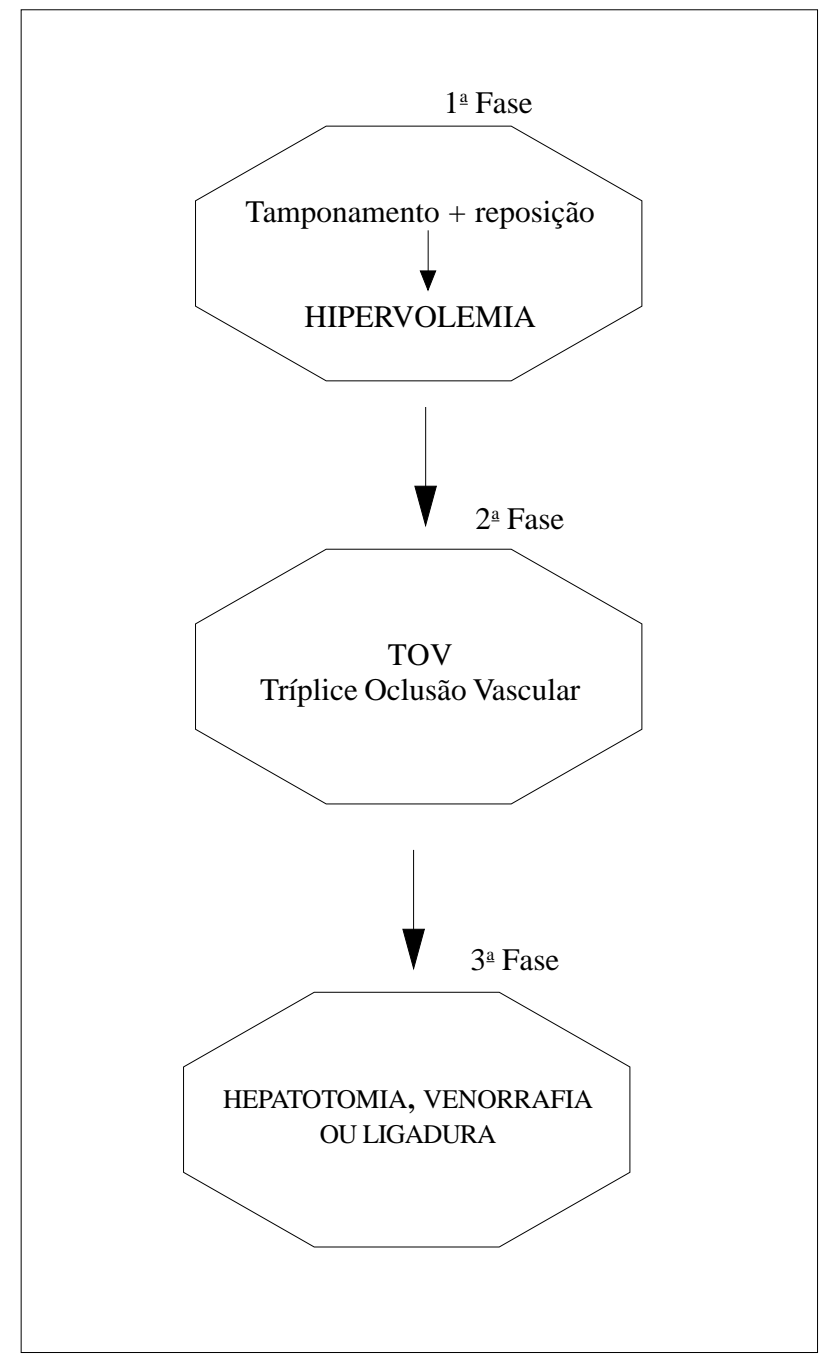

Figura 2 - Fases no tratamento da lesão VCRH e VH e respectivos objetivos.

suscitação" são termos que podem traduzir ou serem entendidos até como condição de volemia normal, mas nunca como estado de hipervolemia, que é a palavrachave na técnica proposta ou a condição fundamental para se evitar as complicações hemodinâmicas da oclusão vascular. É interessante notar que autores que usam expressões vagas recorram com freqüência ou utilizam de rotina a oclusão da aorta para controlar o estado hemodinâmico ${ }^{2}$. Adiantar a volemia com o objetivo de criar hipervolemia é o recurso utilizado para garantir a fase anepática nos transplantes realizados sem auxílio de bomba $^{3}$. Também nas grandes ressecções hepáticas com TOV, a hipervolemia prévia é fator de sucesso do méto$\mathrm{do}^{4}$. É importante enfatizar que em ambas situações existe estabilidade prévia dos pacientes. Nas lesões venosas ocorre o contrario. São pacientes geralmente hipovolêmicos e/ou chocados nos quais a necessidade de hipervolemia para realizar a TOV é tão importante como o reparo da lesão. 


\begin{abstract}
Background: Liver vascular isolation is essential for the treatment of the retrohepatic vena cava and hepatic veins. Triple vessel occlusion (TVO - occlusion of the portal triad, the inferior vena cava above the renal veins and within the pericardium) is the easiest isolation method for the surgeon. Unfortunately, this technique cannot be applied to hypovolemic and/or shock (cardiac arrest) patients as it compromises venous return. Objectives: Our objective is to demonstrate that in the above mentioned patients, establishing a previous hypervolemic state allows the safe use of TVO. Methods: The method includes efficient injury tamponade with aggressive fluid replacement until normal blood volume is reached (resuscitation). Normal blood volume is recognized by a return of arterial blood pressure to normal levels, inferior vena cava filling and an increase in aortic wall tension. Following this procedure, hypervolemia is obtained by the rapid additional infusion of 1.500 to $2.000 \mathrm{ml}$ of fluids. TVO in this situation does not alter the heart rhythm and maintains a clear operative field which is essential for hepatotomy, venorrhaphy and or venous ligation. Results: Three patients were successfully operated.
\end{abstract}

Keywords: Juxtahepatic venous injuries; Hypervolemia; Hepatic vascular isolation; Triple vessel occlusion.

\title{
REFERÊNCIAS
}

1. Hearney JP, Stanton WK, Halbert DS et al.- An improved technique for vascular isolation of the liver. Experimental study and case report. Ann Surg, 1966; 163:237-41.

2. Khaneja SC, Pizzi WF, Barie PS et al. - Management of penetrating juxtahepatic inferior vena cava injuries under total vascular occlusion. J Am Coll Surg, 1997; 184:469-74.
3. Shaw BW, Martin DJ, Marquez JM et al.- Venous bypass in clinical transplantation. Ann Surg, 1984; 200:524-34.

4. Delva E, Barberousse JPB, Nodlinger B et al.- Hemodynamic and biochemical monitoring during major liver resection with use of hepatic vascular exclusion. Surg, 1984; 95:309-18. 